$\beta$-counter, at least 4,000 coincidences at each point being recorded. The genuine coincidence-rate decreased by a factor of about one hundred over the range of absorber values used. There was no trace in the coincidence-absorption curve of the main $600-\mathrm{keV}$. $\beta$-branch in coincidence with the $360 \mathrm{keV}$. $\gamma$-ray, though this, of course, contributes to the accidental coincidences. The absorption curve for the $\beta$-rays of cobalt- 60 under the same conditions was also measured. The two energies were obviously very close, and analysis by Feather's method gives $315 \mathrm{keV}$. for the lower-energy $\beta$-group in iodine-131, a value in excellent agreement with that of Deutsch ${ }^{2}$ based on very much less direct evidence.

Patrick E. Cavanagh

Atomic Energy Research Establishment, Harwell, Berks.

Jan. 29.

1 Harding, Flowers and Eppstein, Nature, 163, 990 (1949). ${ }^{2}$ Metzger and Deutsch, Phys. Rev., 74, 1640 (1948).

\section{Ultra-Violet Band-Systems of CF}

ThERE is little published information about the spectra of the diatomic carbon halides, and only for $\mathrm{CCl}$ has the vibrational analysis of a band-system been completed ${ }^{1,2,6}$. We have, therefore, recently examined some sources which seemed likely to yield a band-spectrum of CF. The most useful of these has proved to be an electrodeless discharge through a stream of fluorocarbon vapour, using a valveoscillator dissipating about 300 watts at a wavelength of about 30 metres. At relatively high pressures, excitation of impurities such as $\mathrm{CO}$ and SiF does not occur, and an extraordinarily rich banded spectrum is obtained, which so far has been found to extend from about 1970 to $4800 \mathrm{~A}$.

The spectrum can be divided into three systems : (i) a system of red-degraded bands forming five close sequences which lie in the region 1970-2210 A. ; (ii) a second ultra-violet system of which the strongest feature is a double double-headed band degraded to the violet at $2240 \mathrm{~A}$.; and (iii) an extensive system of bands not clearly degraded to either direction lying between 2300 and $4800 \mathrm{~A}$.

Of these systems we have so far examined in detail only the two lying at shortest wave-length. The more extensive of these has the heads of its sequences at $1977,2024,2078,2134$ and $2192 \mathrm{~A}$. The assignment of vibrational quantum numbers is not obvious from intensities alone, for the sensitivity of the plates and of the quartz prism spectrographs used varies rather sharply in this region. But from the vibrational analysis, it appears that the $2024 \mathrm{~A}$. sequence is the 0,0 sequence. With this arrangement, about 34 bands have been assigned to a scheme represented by the following equation :

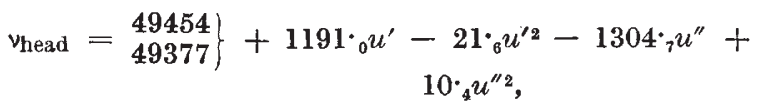

where $u=v+\frac{1}{2}$. Most of these bands are accom. panied by somewhat weaker heads lying at a distance of between 20 and $60 \mathrm{~cm}^{-1}$ to higher wave-number; this distance varies with vibrational quantum number in the way expected for a head-origin separation.

The violet-degraded band at $2240 \mathrm{~A}$. is strikingly similar in appearance to the $\gamma$-bands $\left(2 \Sigma-{ }^{2} \Pi\right)$ of NO. Its herds are at $44,679 \cdot 0,44,666 \cdot 2,44,604 \cdot 1$ and $44,585 \cdot 2 \mathrm{~cm}^{-1}$. The separation of the inner heads, $74 \cdot{ }_{9} \mathrm{~cm}^{-1}$, is very close to the electronic interval $\left(77 \mathrm{~cm}^{-1}\right)$ found from the analysis of the other system above, and suggests that these systems may have a state in common. This view is supported by the observation of weaker violet-degraded heads at about $2304 \cdot 0$ and $2308 \cdot 2 \mathrm{~A}$., which are at about the right distances from the heads of the $2240 \mathrm{~A}$. band (which is probably the 1,0 band) to be the 1,1 heads with a lower-state vibrational interval equal to that found for the lower state of the first system.

It seems likely that the lower state of these two systems is the ground-state of CF $\left({ }^{2} i 1\right)$. The multiplet separation, about $75 \mathrm{~cm}^{-1}$, is of the order expected from that observed ${ }^{2,6}$ in $\mathrm{CCl}\left(96 \mathrm{~cm}^{-1}\right)$ and is not far from the value $60 \mathrm{~cm}^{-1}$ predicted by Howell ${ }^{3}$. The force-constant, $k_{e}{ }^{\prime \prime}, 7.37 \times 10^{5} \mathrm{dyne} / \mathrm{cm}$., is also of the order expected by analogy with other halides, thus :

$$
\begin{array}{llllll}
\mathrm{BeF} & 5 \cdot 77 & \mathrm{BF} & 8 \cdot 05 & \mathrm{CF} & 7 \cdot 37 \\
\mathrm{BeCl} & 3 \cdot 03 & \mathrm{BCl} & 3 \cdot 47 & \mathrm{CCl} & 3 \cdot 93
\end{array}
$$

We are indebted to Messrs. I.C.I. (General Chemicals), Ltd., for the fluorocarbon used in these experiments.

Notes added on April 6. A rotational analysis of the 2240 band has now been made. This shows that it does indeed arise from $a^{2} \Sigma-{ }^{2} \Pi$ transition, with $B^{\prime \prime} \sim 1.41, B^{\prime} \sim 1.69 \mathrm{~cm} .^{-1}$. The value of $r_{0}{ }^{\prime \prime}$ for $\mathrm{CF}$ is thus about $1 \cdot 27_{5} \mathrm{~A}$.

A vibrational analysis of system (iii) has recently been given by Venkateswarlu ${ }^{5}$, who attributes it to non-linear $\mathrm{CF}_{2}$.

E. B. Andrews

R. F. BARROW

Physical Chemistry Laboratory,

University of Oxford.

$$
\text { Jan. } 15 .
$$

${ }^{1}$ Asundi and Karim, Proc. Ind. Acad. Sci., 6A, 328 (1937).

2 Horie, Proc. Phys, Math. Soc., Japan, 21, 143 (1939).

${ }^{3}$ Howell, Proc. Phys. Soc., 57, 37 (1945).

$₫$ Chrétien and Miescher, Nature, 163, 996 (1949).

s Phys. Rev. (March 1950).

- Recent work by Venkateswarlu on CCl, Phys. Rev., 77, 79 (1950), leads to somewhat different values of the constants than those of $\mathrm{Horie}^{2}$ which are used here, but the qualitative arguments are unaffected.

\section{Production of an Irreversible Joshi Effect in the Hysteresis-Range below the Threshold Potential}

THE fundamental importance of the threshold potential $V_{m}$ in the production of the Joshi effect $\Delta i$ and the preferential incidence of the latter in the high-frequency components of the discharge current have been established ${ }^{1-6}$. Results were suggestive when $\Delta i$ was studied in chlorine at and below $V_{m}$. Until this latter is reached, the current is small, say, $i_{1}$; it increases suddenly by a large amount at $V_{m}$, and rises rapidly thereafter. On decreasing the voltage below $V_{m}$, it decreased gradually to $i_{1}$ at $V_{1}$ (see graph). $C A$ denotes $i_{D}$, the current in the dark when the voltage is reduced below $V_{m}$, and $D E$ the corresponding current $i_{L}$ under irradiation. The larger current (in the dark) while decreasing below the voltage $V_{m}$ is due to hysteresis.

The Joshi effect below $V_{m}$ increased progressively to almost 100 per cent current diminution in light at a certain voltage, say, $V_{R}$, and continued to be so up to $V_{1}$. Within this range of voltage $V_{R}-V_{1}$, it was remarkable to observe that the photo-suppression of $i$ and also of the associated high frequencies, con- 